\title{
Particle Based Model of Tumor Progression Stimulated by the Process of Angiogenesis
}

\author{
Rafał Wcisło and Witold Dzwinel \\ AGH University of Science and Technology, Institute of Computer Science \\ al. Mickiewicza 30, 30-059 Kraków, Poland \\ wcislo@agh.edu.pl
}

\begin{abstract}
We discuss a novel metaphor of tumor progression stimulated by the process of angiogenesis. The realistic 3-D dynamics of the entire system consisting of the tumor, tissue cells, blood vessels and blood flow can be reproduced by using interacting particles. The particles mimic the clusters of tumor cells. They interact with their closest neighbors via semi-harmonic forces simulating mechanical resistance of the cell walls and the external pressure. The particle dynamics is governed by both the Newtonian laws of motion and the rules of cell life-cycle. The particles replicate by a simple mechanism of division, similar to that of a single cell reproduction and die due to necrosis or apoptosis. We conclude that this concept can serve as a general framework for designing advanced multi-scale models of tumor dynamics. In respect to spatio-temporal scale, the interactions between particles can define e.g., cluster-to-cluster, cellto-cell, red blood cells and fluid particles interactions, cytokines motion, etc. Consequently, they influence the macroscopic dynamics of the particle ensembles in various sub-scales ranging from diffusion of cytokines, blood flow up to growth of tumor and vascular network expansion.
\end{abstract}

Keywords: tumor progression, angiogenesis, computer simulation, particle model.

\section{Introduction}

Angiogenesis is a process of the blood vessel formation from a pre-existing vasculature $[1,2]$. It refers to complex biological phenomena that appear at various scales $[3,4]$. They ranged from cellular scale to the macroscopic scale corresponding to concentration of cancer cells into compact clusters. The vessel sprouts are formed in response to cytokines produced by the tumor cells in hypoxia. Initially, they develop mainly due to endothelial cell migration. Finally, they organize themselves into a branched, connected network, which supply nutrients (oxygen) to the tumor cells. Tumor-induced angiogenesis provides the fundamental link between the avascular phase of tumor growth and the more invasive vascular phase. Vascularized tumor attacks the surrounding tissue and blood system and the possibility of the cancer spreading (metastasis) increases dramatically.

Judah Folkman published in 1971 the theory [1] that angiogenesis is a principal process in tumor progression. The clinical importance of angiogenesis as a prognostic tool is now well understood [2]. Since tumor dynamics depends on angiogenesis, 
there is strong interest in using antiangiogenic drugs to treat cancer [5]. The process of angiogenic signaling and formation of blood vessels can be disrupted or slowed down with small molecules. In addition to treatments directed to specific target, non specific agents can be used to eliminate endothelial cells thus inhibiting angiogenesis. This involves numerous expensive and arduous investigations both of hundreds of factors inhibiting angiogenesis and antiangiogenic chemical species which can be considered in drug design process. To cut expenses, the predictive power of mathematical modeling and computer simulation has to be employed. As shown in [6], in silico experiments can play the role of angiogenesis assays.

Mathematical modeling of angiogenesis extends back a number of years [3-11]. The modeling concentrates on key events such as the response of endothelial cells to tumor angiogenic factors (TAF) secreted by a solid tumor, endothelial cell proliferation, endothelial cell interactions with extracellular matrix macromolecules, capillary sprout branching and vessel maturation. The substantial difference with respect to the physical models (such as the kinetic theory) is that the microscopic state of the cells is defined not only by mechanical variables, such as position, velocity, pressure, but also by internal biological microscopic phenomena reflecting activities of the cells. Several models $[4,6,7]$ have used techniques based on the partial differential equations (PDE) to examine the functions in space and time of endothelial cell density, cytokine concentration, capillary tip and branch density. Nevertheless, within these models, it is not possible to capture such the important events as repeated sprout branching and the overall dendritic structure of the network. In contrast to these deterministic, continuum models, several different types of discrete techniques, such as: cellular automata and DLA [8], L-systems [9], and level-set method [10], are being used. Unlike in the continuum models, discrete models can follow individual cells and can reveal more details about cell dynamics and its interaction with the tissue. As shown in $[3,4,6]$, the results of discrete models agree well with the predictions of the continuum model. In addition they are able to produce capillary networks with topology and morphology qualitatively similar to those observed in experiments.

However, any of these techniques cannot be considered as flexible framework for developing advanced multi-scale models. The extension of CA to more general complex automata paradigm, e.g., as shown in [11], is rather an exception. Moreover, the difficulties in modeling tumor progression in its natural environment involving mechanical forces from surrounding environment and continuous remodeling of vessel networks in various types of tissue (muscle, bones, lungs) is a serious drawback of this on-grid methods. Other important aspects, lacking in the most of models of angiogenesis, are related to the blood flow through the capillary network. The blood flow has important consequences for drug delivery and optimization of chemotherapy regimes. Recent work modeling the flow through vascular networks appears in [12].

We discuss here the advantages of the moving particles model [13] as a metaphor of the process of tumor dynamics stimulated by the angiogenesis and as a useful framework for more advanced multi-scale models. In the following sections we describe the model of tumor progression and its particle based realization. Finally, we present the conclusions and discuss possible extensions of the model. 


\section{Simplified Model of Tumor Growth}

The simplified model (basing on $[3,4,6]$ ) of tumor progression can be described in the three overlapping phases of growth: the avascular phase (I), the angiogenic phase (II) and the vascular phase (III).

Phase I. Solid tumor, which is smaller than $2 \mathrm{~mm}$ in diameter, removes waste and acquires nutrients and oxygen through passive diffusion. The oxygen (nutrients), supplied by nearby blood vessels, filter through the surface of the solid tumor and diffuse in its body. The tumor cluster consists of the outer region with proliferating cells, an intermediate region of cells in hypoxia and the necrotic core of dead tumor cells. The cells in hypoxia produce cytokines called TAF (tumor angiogenic factors) such as growth inhibitory factors (GIF), and growth promoting factors (GPF). The GIF and GPF concentrations influence the tumor cells mitotic rate.

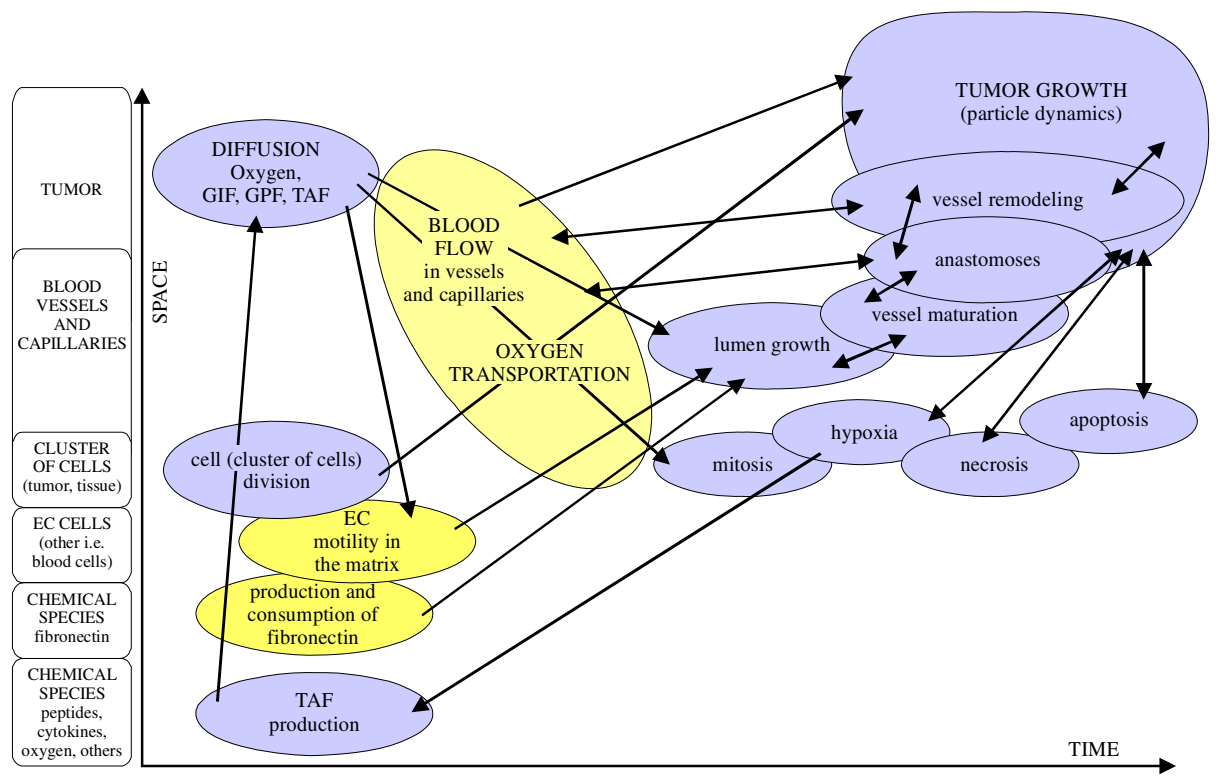

Fig. 1. The scale separation map of the main processes considered in tumor-induced angiogenesis

Phase II. TAF diffuse through the tissue (extracellular matrix) thereby creating chemical gradients in the body surrounding the tumor. Once the cytokines reach any neighboring blood vessels its critical concentration stimulates several enzymes to degrade of basement membrane. Simultaneously extracellular matrix is degraded and endothelial cells migrate toward the growth factor sources and proliferate (chemotaxis). The fingering capillary sprouts are formed then by accumulation of endothelial cells which are recruited from the parent vessel. Due to the migration and further recruitment of endothelial cells at the sprout-tip the sprouts grow in length and continue to move towards the tumor. The high-molecular-weight glycoproteins - fibronectin - promote cell 
migration up its concentration gradient (haptotaxis). Initially, the sprouts grow parallel to each other. Then they tend to incline toward each other [5]. This leads to numerous tip-to-sprout links known as anastomoses. A network of loops or arcades develops. The blood starts to circulate in newly developed vessels. From the primary loops, new sprouts emerge providing for the further extension of the new capillary bed.

Phase III. Once the sprouts approach the tumor, their branching dramatically increases until the tumor is eventually penetrated by vascular network. Due to better oxygenation the concentration of TAF decreases also inside the tumor. However, the newly formed vessels are subsequently remodeled due to tumor growth and pushed away producing regions of lesser concentration of oxygen, which initiates TAF production. This causes simultaneous growth in size of both tumor and its vasculature.

In Fig. 1 we present the scale separation map (SSM) for this simplified model of tumor growth. The macroscopic scale refers to phenomena, which are typical for continuum systems: diffusion (oxygen, TAF), overall tumor condensation, blood flow while microscopic processes such as cell motility, cell reproduction (division), cell death (necrosis and apoptosis) are discrete. The arrows show dependences between these processes. The particle model presented in the following section refers only to the processes shaded in dark.

\section{Particle Model}

In Fig. 2 we show the building blocks of our model while in Fig. 3 its implementation is briefly described. Unlike the simulations reproducing this process outside the tumor $[3,4,6]$, we assume that the vasculature develops inside the growing cluster. This lets us to simulate the combination of Phases II and III and show how our model will cope with vasculature remodeling caused by mechanical forces being the result of the pressure exerted by the swelling tumor on the expanding vascular network.

The particle model of angiogenesis follows the general principles of particle model construction described in [13]. The particles mimic the clusters of tumor cells. The cluster size depends on the model granularity. Particularly, in the finest spatial scale level, it can represent a single tumor cell. Nevertheless, this is rather unrealistic assumption, having in mind that the tumor of $1 \mathrm{~mm}$ in diameter consists of hundred of millions of cells. The tumor particles interact with each other via forces, which mimics mechanical interactions of the cell walls. In more advanced models surrounding tissue cells can be represented by using different kind of particles (e.g. by defining different interaction potential). Instead, we assume that only the tumor cells exist while the external pressure is simulated by the attractive tail of asymmetric interparticle potential $\Omega\left(d_{i j}\right)$ defined as follows:

$$
\Omega\left(d_{i j}\right)=\left\{\begin{array}{l}
a d_{i j}{ }^{2}, \text { for } d_{i j}<d_{c u t}, \\
a_{2} d_{c u t}{ }^{2}, \text { for } d \geq d_{c u t}
\end{array} \text { where } a=\left\{\begin{array}{l}
a_{1} \text { for } d<0 \\
a_{2} \text { for } d \geq 0
\end{array} \text { and } a_{1}<<a_{2}\right.\right.
$$




$$
d_{i j}=\left|\vec{r}_{i j}\right|-\left(r_{i}+r_{j}\right)
$$

where $d_{i j}$ is the distance between particles. The particle interactions are depicted in Fig. 4.

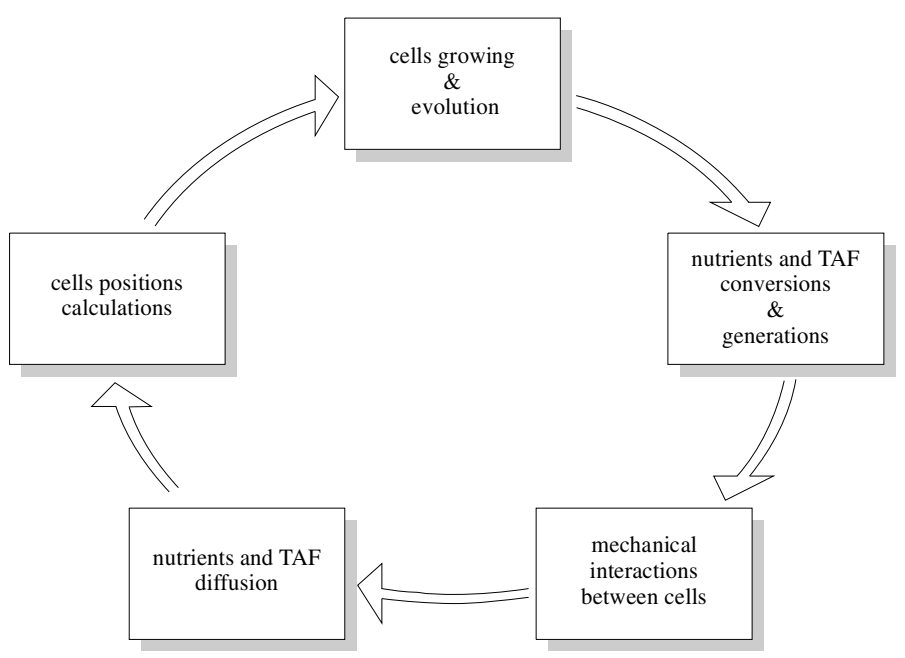

Fig. 2. Building blocks of the tumor-induced angiogenesis model

The particle motion is governed by the Newtonian laws, that is:

$$
m_{i} \frac{d \mathbf{V}_{i}}{d t}=-a \cdot \nabla \Omega\left(d_{i j}\right)-\lambda \cdot \mathbf{V}_{i} \quad \frac{d \mathbf{r}_{i}}{d t}=\mathbf{V}_{i} \quad r_{i j}=\left(\mathbf{r}_{i}-\mathbf{r}_{j}\right) \cdot\left(\mathbf{r}_{i}-\mathbf{r}_{j}\right)^{T}
$$

where $\mathbf{r}_{i}$ and $\mathbf{V}_{i}$ are the position and velocity of particle $i$, respectively, while $\lambda$ is the friction coefficient.

The diffusion of oxygen and TAF is faster than the process of tumor growth. Therefore, the concentrations of TAF and oxygen can be computed in the steady state. This allows for using the fast procedure of solving Laplace equation on a discrete irregular lattice of particles. Only the vessels with circulating blood (anastomosing vessels) are the sources of oxygen while the cells in hypoxia are the sources of TAF. In our simplified model we neglect blood flow in capillaries [5]. We assume instead, that all the vessels are sources of oxygen. Because the blood circulation is slower than diffusion but faster than mitosis cycle [6], in the following version of the model, the flow intensity in anastomozing vessels will be computed on the base of Kirchhoff's laws [5].

The microscopic processes of the cell life-cycle and the growth of vascular network are simulated by using the following rules of tumor growth and blood vessel network expansion. 


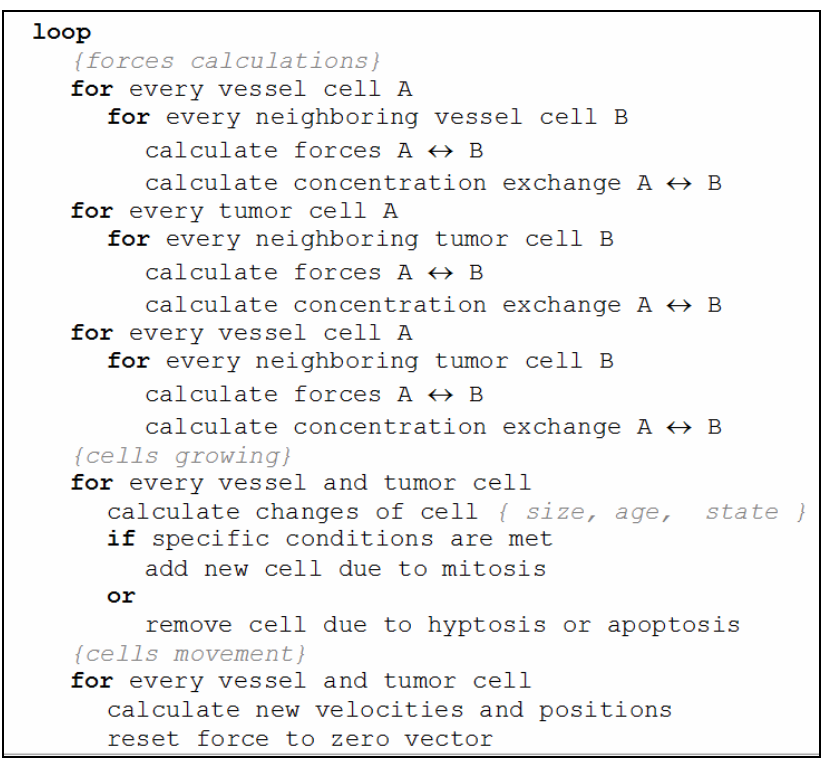

Fig. 3. The implementation scheme of the particle model of angiogenesis
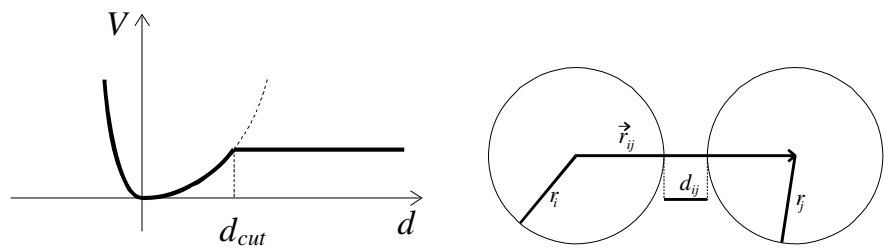

Fig. 4. The shape of inter-particle potential representing mechanical interactions between cell clusters

Tumor Growth. During the life-cycle the cluster of tumor cells (the particle) changes its state from new to necrotic, according to its individual clock and oxygen concentration. The particles of a given age and size being well oxygenated undergo the process of "mitosis", i.e., one particle (the "mother" particle) divides producing two daughter particles. New born particle is in new state and has diameter equal to $d_{M I N}$. It grows with time, proportionally to oxygen concentration up to $d_{M A X}$. The minimum and maximum particle diameters depend on the model granularity. Finally, after time $\boldsymbol{T}_{N}$, the particles undergo apoptosis, i.e., programmed cell depth. The dead particles are in necrotic state and are removed from the system. For oxygen concentration smaller than a given threshold, the particle state (excluding necrotic one) can be converted to hypoxia state becoming the source of TAF. The particles, which are in hypoxia for a period of time longer than a given threshold, die (necrosis) and are removed from the system. 
Expansion of Blood Vessel Network. The blood vessels are made also of particles. The new sprout is formed when the TAF concentration exceeds a given threshold. Then the "vessel particle" undergoes the process of "mitosis" directed to the local gradient of TAF concentration. The haptotaxis is not included yet in the model. Only particles close to the sprout tip can replicate. The interactions between both two identical vessel particles and vessel_particle-tumor_particle describe the same potential as defined by Eq.1.
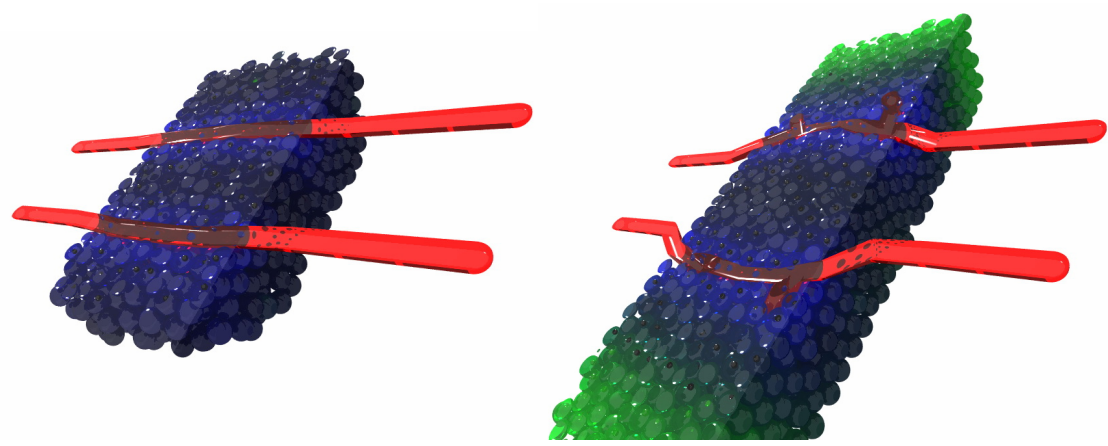

Fig. 5. Mechanical remodeling - growing tumor pushes the arteries away

Unlike in simulations of the angiogenesis described in $[4,6]$, where the vascularization starts and develops outside the tumor, our model allows the growth of tumor simultaneously with its internal vascularization. This situation is much more complicated, mainly due to the changes in TAF production and mechanical forces involving blood vessels remodeling. In Fig. 4 we display two snapshots of simulation, which shows how the growing tumor pushes the arteries away.
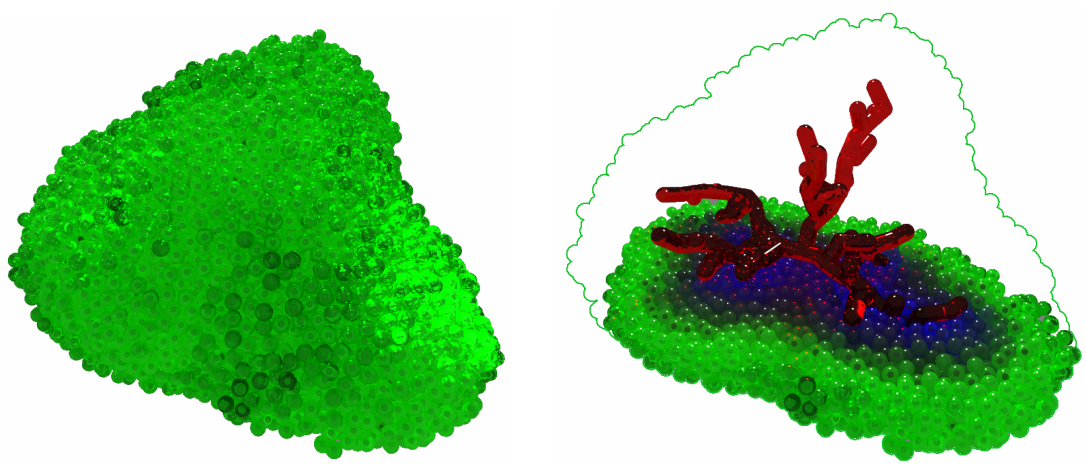

Fig. 6. The snapshot from 3-D simulation of tumor growth and the vessels formation due to the process of angiogenesis. The cells shaded in dark are better oxygenated. 


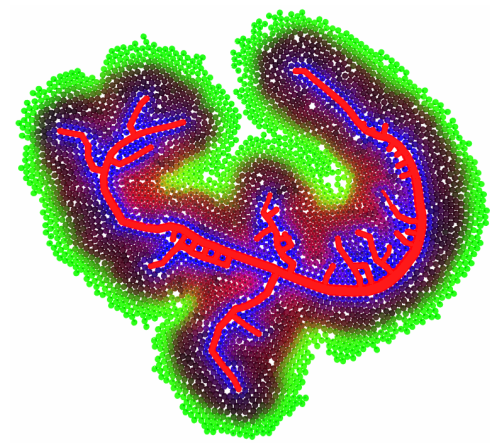

Fig. 7. Snapshot from 2-D simulations showing simultaneous growth of tumor and its vascular network. Darker regions display well oxygenated tumor cells.

We started the simulations with a single "vessel particle" located in the centre of small tumor cluster. Of course, this situation is not realistic. The initial conditions should consist of avascular tumor cluster located close to the blood capillary, which produces the network of capillaries growing towards the tumor.

Moreover, only anastomosing vessels allowing for blood circulation are the new sources of oxygen while in our simulations all the vessel particles are the sources of oxygen. However, this is only the technical problem, which can be solved in the nearest future. The Figs. 6, 7 display the unique possibility of the particle based model, which can simulate tumor progression simultaneously with vascular network continuously remodeled by mechanical forces exerted by the tumor body.

\section{Concluding Remarks}

Our concept of interacting particles represents a new framework for a more precise multi-scale model of the intrinsically complex process of angiogenesis. This approach is competitive to existing paradigms employing heterogeneous sub-models [4] or cellular automata $[8,11]$. The possibility of simulation of progression of vascularized tumor involving simultaneous remodeling of the blood vessel system is the great advantage of particle based approach over other approaches. Moreover, realistic 3-D dynamics of the entire system consisting of the tumor and other tissue cells, blood vessels and blood flow can be reproduced by using the same model. The scalability of the particle model allows for simulating of large systems consisting of hundred of millions of particles.

Our model can be supplemented by other microscopic and macroscopic processes, e.g., by modifying the "interaction" forces between clusters, incorporating more precise models of reproduction mechanism, cell-life cycle, lumen growth dynamics, blood flow in the vessels, including haptotaxis mechanism and others. The model can be supplemented also by such important process like blood circulation. In [14] we show that blood flow can be modeled by using dissipative particle dynamics in a very short spatio-temporal scale [14] (see Fig. 8). 


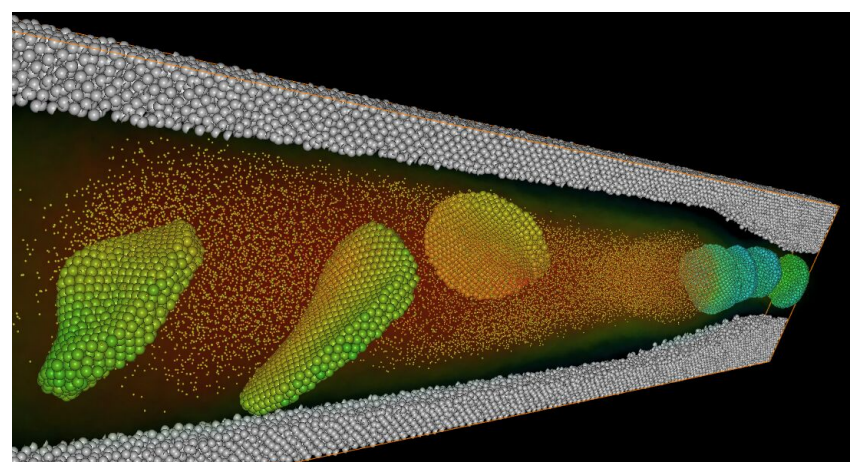

Fig. 8. The snapshot from simulation of red blood cells flowing in the vain with the chocking point modeled using fluid particle model [14]

Currently, we are working on using smoothed particle hydrodynamics (SPH) employing viscoelastic rheological models (like the Casson fluid) for simulating blood flows in larger scales. These two models of the processes of tumor growth and blood flow are planned to be combined together (see Fig. 1) in the scope of generalized multiscale particle model.

The particle model of blood flow can be fine grained employing large number of particles $\left(>10^{6}\right)$. As we show in [15], the code can be easily parallelized and run on multiprocessor cluster to speed up computations. We have estimated that we could obtain at least the same speed-up factor (i.e., about 20 on 32 processors) for the model of tumor growth.

We plan to validate the model on the basis of the comparison between realistic images of tumor vascular networks from confocal microscopy and computer experiments. The comparisons will be made employing structural properties of tumor vascularization. The vascular networks can be described by the feature vectors with statistical and/or algebraic descriptors of complex networks as the vector components. Finally, pattern recognition methods such as clustering and features extraction will be used for data classification.

Acknowledgments. We are indebted to Professor dr Arkadiusz Dudek from the University of Minnesota Medical School for numerous comments, valuable suggestions and his overall contribution to this work. This research is financed by the Polish Ministry of Education and Science, Project No. 3 T11F 01030.

\section{References}

1. Folkman, J.: Tumor angiogenesis: Therapeutic implications. N. Engl. J. Med. 285, 11821186 (1971)

2. Ferrara, N., Chen, H., Davis Smyth, T., Gerber, H.P., Nguyen, T.N., Peers, D., Chisholm, V., Hillan, K.J., Schwall, R.H.: Vascular endothelial growth factor is essential for corpus luteum angiogenesis. Nat. Med. 4, 336-340 (1998) 
3. Mantzaris, N., Webb, S., Othmer, H.G.: Mathematical Modeling of Tumor-induced Angiogenesis. J. Math. Biol. 49(2), 1416-1432 (2004)

4. Bellomo, N., de Angelis, E., Preziosi, L.: Multiscale Modeling and Mathematical Problems Related to Tumor Evolution and Medical Therapy. J. Theor. Med. 5(2), 111-136 (2003)

5. Stéphanou, A., McDougall, S.R., Anderson, A.R.A., Chaplain, M.A.J., Sherratt, J.A.: Mathematical Modelling of Flow in 2D and 3D Vascular Networks: Applications to Antiangiogenic and Chemotherapeutic Drug Strategies. J. Math. Comput. Model 41, 11371156 (2005)

6. Chaplain, M.A.J.: Mathematical modelling of angiogenesis. J. Neuro-Oncol. 50, 37-51 (2000)

7. Luo, S., Nie, Y.: FEM-based simulation of tumor growth in medical image. In: Galloway Jr., R.L. (ed.) Medical Imaging 2004: Visualization, Image Guided Procedures, and Display. Proceedings of SPIE, vol. 5367, pp. 600-608 (2004)

8. Moreira, J., Deutsch, A.: Cellular Automaton Models of Tumor Development: A Critical Review. Adv. Complex Syst. 5/2(3), 247-269 (2002)

9. Fracchia, F.D., Prusinkiewicz, P., de Boer, M.J.M.: Animation of the development of multicellular structures. In: Magnenat-Thalmann, N., Thalmann, D. (eds.) Computer Animation 1990, pp. 3-18. Springer, Tokyo (1990)

10. Lappa, M.: A CFD level-set method for soft tissue growth: theory and fundamental equations. J. Biomech. 38(1), 185-190 (2005)

11. Hoekstra, A.G., Lorenz, E., Falcone, L.-C., Chopard, B.: Towards a Complex Automata Framework for Multi-scale Modeling: Formalism and the Scale Separation Map. In: Shi, Y., van Albada, G.D., Dongarra, J., Sloot, P.M.A. (eds.) ICCS 2007. LNCS, vol. 4487, pp. 1611-3349. Springer, Heidelberg (2007)

12. McDougall, S.R., Anderson, A.R.A., Chaplain, M.A.J., Sherratt, J.A.: Mathematical modelling of flow through vascular networks: Implications for tumour-induced angiogenesis and chemotherapy strategies. Bull. Math. Biol. 64, 673-702 (2002)

13. Dzwinel, W., Alda, W., Yuen, D.A.: Cross-Scale Numerical Simulations Using DiscreteParticle Models. Molecular Simulation 22, 397-418 (1999)

14. Dzwinel, W., Boryczko, K., Yuen, D.A.: A Discrete-Particle Model of Blood Dynamics in Capillary Vessels. J. Colloid Int Sci. 258(1), 163-173 (2003)

15. Boryczko, K., Dzwinel, W., Yuen, D.A.: Modeling Heterogeneous Mesoscopic Fluids in Irregular Geometries using Shared Memory Systems. Molecular Simulation 31(1), 45-56 (2005) 